\title{
All-optical injection of ballistic electrical currents in unbiased silicon
}

\author{
LOUIS COSTA, MARKUS BETZ, MARKO SPASENOVIĆ, ALAN D. BRISTOW AND HENRY M. VAN DRIEL* \\ Department of Physics and Institute for Optical Science, University of Toronto, Toronto M5S 1A7, Canada \\ *e-mail: vandriel@physics.utoronto.ca
}

Silicon has been the dominant material in electronics since the invention of the integrated transistor. In contrast, silicon's indirect bandgap and vanishing second-order optical nonlinearity limit its applications in optoelectronics ${ }^{1}$. Although all-optical components such as Raman lasers ${ }^{2}$, parametric amplifiers $^{3}$ and electro-optic modulators ${ }^{4,5}$ have recently been reported, control over charge motion in silicon has only ever been achieved electronically. Here, we report all-optical generation of ultrafast ballistic electrical currents in clean, unbiased, bulk silicon at room temperature. This current injection, which provides new insights into optical processes in silicon, results from quantum interference between one- and twophoton absorption pathways across the indirect bandgap despite phonon participation and the multi-valley conduction band. The transient currents induced by $150 \mathrm{fs}$ pulses are detected via the emitted $\mathrm{THz}$ radiation. The efficiency of this third-order optical process is surprisingly large for fundamental wavelengths in the $1,420-1,800 \mathrm{~nm}$ range.

The use of phase-related optical beams has advanced the application of light in a variety of processes such as controlling photochemical reactions ${ }^{6}$, forming attosecond pulses ${ }^{7}$ and producing high-intensity $\mathrm{THz}$ pulses in air ${ }^{8}$. In directbandgap semiconductors, both charge ${ }^{9,10}$ and pure spin currents ${ }^{11,12}$ have been generated through quantum interference of singleand two-photon interband absorption of light at frequencies $2 \omega$ and $\omega$ with the current dependent on the phase parameter $\Delta \phi=2 \phi_{\omega}-\phi_{2 \omega}$; here $\phi_{\omega, 2 \omega}$ is the phase of the optical beam. For electrical-current generation in $\mathrm{Si}$, we use $150 \mathrm{fs}$ pulses with $0.69<\hbar \omega<0.87 \mathrm{eV}(1,420-1,800 \mathrm{~nm}$ wavelength). Both single-photon absorption at $2 \omega$ and two-photon absorption at $\omega$ occur across the indirect bandgap ${ }^{13}, 1.1 \mathrm{eV}$, but below the direct bandgap, $3.5 \mathrm{eV}$. Spatially and temporally overlapped collinearly polarized $\omega$ and $2 \omega$ pulses are focused at normal incidence on (001)-oriented crystalline silicon samples of thickness between 0.5 and $500 \mu \mathrm{m}$. The different samples produce similar results and here we mainly consider results from a $125-\mu \mathrm{m}$-thick wafer. With $\hbar \omega=0.8 \mathrm{eV}$ and a fundamental (second harmonic) pulse energy of $120 \mathrm{~nJ}(4 \mathrm{~nJ})$, the near-surface carrier densities generated by each pulse are $5 \times 10^{15} \mathrm{~cm}^{-3}\left(6 \times 10^{16} \mathrm{~cm}^{-3}\right)$ for a singlephoton absorption coefficient ${ }^{13}$ of $1.3 \times 10^{3} \mathrm{~cm}^{-1}$ and two-photon absorption coefficient $\mathrm{t}^{14,15}$ of $0.45 \mathrm{~cm} \mathrm{GW}^{-1}$. The transient electrical current, directed in the plane of the sample, emits $\mathrm{THz}$ radiation that is detected by electro-optic sampling.

Figure 1a shows the $\mathrm{THz}$ field as a function of time delay between $0.8 / 1.6 \mathrm{eV}$ pump pulses and an electro-optic sampling $1.53 \mathrm{eV}$ probe pulse; traces are shown for values of $\Delta \phi$ separated by $\pi$. The oscillatory structure reflects the narrow bandwidth of the electro-optic detection system. The actual time-resolved currents, as revealed by larger bandwidth detection, relax in $<1 \mathrm{ps.} \mathrm{The}$ peak $\mathrm{THz}$ amplitude is $\sim 10 \mathrm{~V} \mathrm{~m}^{-1}$, corresponding to an estimated current density of $0.5 \mathrm{kA} \mathrm{cm}^{-2}$ (see ref. 16 and the Methods section). Figure la shows that the $\mathrm{THz}$ field reverses sign when $\Delta \phi$ changes by $\pi$, consistent with a coherence-induced current source. A more detailed dependence of the $\mathrm{THz}$ field on $\Delta \phi$ is shown in Fig. 1b; the sinusoidal dependence of the $\mathrm{THz}$ field on $\Delta \phi$ is also consistent with the current-injection process. The current amplitude and scalar $(+/-)$ direction can be controlled through the phase parameter, $\Delta \phi$, alone. The current vector can be controlled by redirecting the beam polarization.

Figure 2 shows how the amplitude of the $\mathrm{THz}$ field/current density varies with average pump power, $P^{\omega, 2 \omega}$, and $2 \hbar \omega$. In Fig. $2 \mathrm{a}$, the $\mathrm{THz}$ field is seen to vary as $P^{\omega} \sqrt{P^{2 \omega}}$, consistent with a third-order, $\chi^{(3)}$, nonlinear optical process. Measurements on $\mathrm{Si}$ samples of different thickness give similar phase and power scaling behaviour. However, for the $125-\mu \mathrm{m}$-thick sample, the $\mathrm{THz}$ field is a factor of $\sim 3$ smaller than that for the $2 \mu \mathrm{m}$ layer, owing to phase walk-off between the $\omega$ and $2 \omega$ pulses (refractive indices of 3.37 and 3.71, respectively). This induces periodic current reversal over the $\sim 15 \mu \mathrm{m}$ optical-field attenuation depth and a smaller total current than in an optically thin sample. Although the $2-\mu \mathrm{m}-$ thick sample absorbs only $\sim 10 \%$ of the incident light, its thickness is $2 / 3$ of the coherence length of the optical mixing process. Figure $2 \mathrm{~b}$ shows the dependence of the current-injection efficiency on $2 \hbar \omega$. For comparison purposes, data are also shown for the $\mathrm{THz}$ signals emitted by a $1-\mu \mathrm{m}$-thick (001) GaAs and a 1- $\mu \mathrm{m}$-thick (001) InP sample (each thickness being comparable to the singlephoton absorption depth) illuminated under identical conditions. For small $2 \hbar \omega$, the dispersion is consistent with the onset of current injection near the fundamental bandgap $(1.42 \mathrm{eV}$ for GaAs, $1.34 \mathrm{eV}$ for InP). For larger $2 \hbar \omega$, the injected current is almost constant beyond $1.5 \mathrm{eV}$, probably related to the counterbalancing increasing density of optically coupled states and a decreasing momentum scattering time.

The current's phase and optical power dependence suggests a $\chi^{(3)}$ nonlinear process. Interference of singleand two-photon absorption is known to inject an electrical current density in direct-bandgap materials according to ${ }^{10}$ $\mathrm{d} J_{i}^{i n j} / \mathrm{d} t=2 \eta_{i j k l} E_{j}^{\omega} E_{k}^{\omega} E_{l}^{2 \omega} \sin \Delta \phi$. Here, $\eta_{i j k l}$ is a fourth-rank tensor (related to a divergent, imaginary part of a third-order optical susceptibility, $\left.\operatorname{Im} \chi^{(3)}(0 ; \omega, \omega,-2 \omega)\right)$ and $E^{\omega, 2 \omega}$ is the field envelope of the $\omega, 2 \omega$ pulses. Following injection, the current should evolve via space-charge effects and carrier momentum 

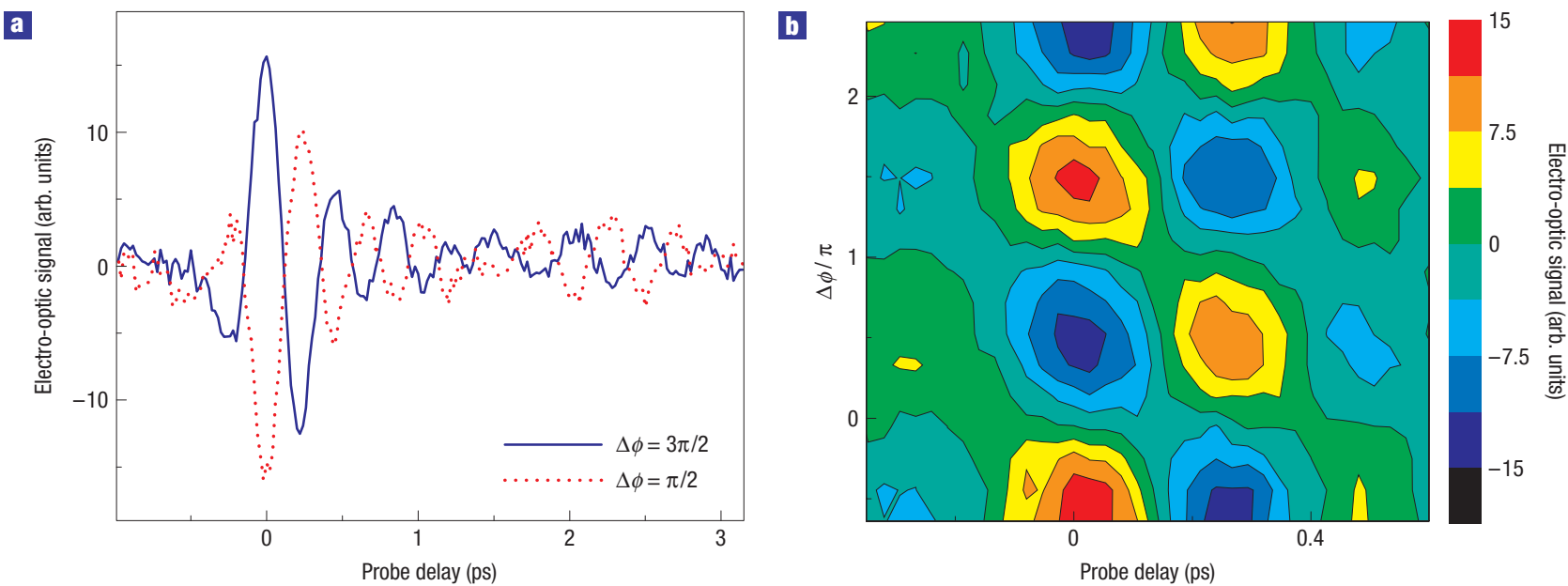

Figure 1 Temporal and phase dependence of THz emission from injected currents for $\mathbf{0 . 8}$ and $\mathbf{1 . 6} \mathbf{~ e V}$ pump pulses. a, Time-dependent electro-optic signals of THz fields from a 125- $\mu \mathrm{m}$-thick Si wafer for different values of $\Delta \phi$. $\mathbf{b}$, Two-dimensional contour plot of THz amplitude as a function of pump-probe delay and $\Delta \phi$.
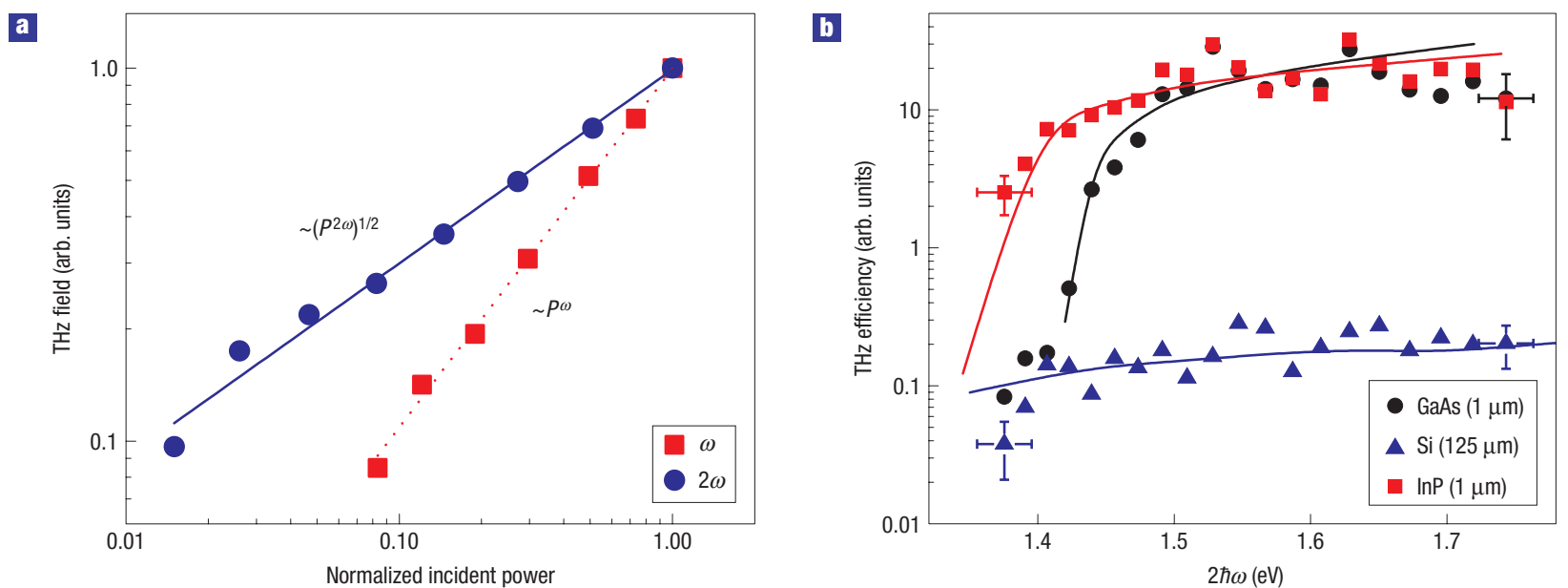

Figure 2 Dependence of THz emission on pump power and photon energy. a, THz amplitude from a 125- $\mu$ m-thick silicon sample as a function of the average power of one of the co-linearly polarized pump beams when the other pump beam's average power is fixed. For ease of display, the powers are normalized to yield the same maximum THz field at the highest power. The lines indicate linear and square-root power laws. b, THz field strengths obtained from (001) GaAs, InP and Si crystals of indicated thickness as a function of photon energy $2 \hbar \omega$. The field strengths are normalized by $P^{\omega}\left(P^{2 \omega}\right)^{1 / 2}$ to account for variation in pump power with tuning. The solid curves are predictions of the dispersion on the basis of the one- and two-photon absorption coefficients of the different materials. The horizontal and vertical error bars indicate the energy bandwidth of the excitation pulses and the standard deviation of the THz signal strength, respectively.

relaxation. The experimental data are also consistent with a $\operatorname{Re} \chi^{(3)}(0 ; \omega, \omega,-2 \omega)$ process, such as (non-resonant) optical rectification. However, as dispersion of $\operatorname{Re} \chi^{(3)}$ is expected to be small for $\hbar \omega$ well below the direct bandgap, for our photon energy, $\operatorname{Re} \chi^{(3)}(0 ; \omega, \omega,-2 \omega)$ can be estimated either by its low-frequency limit ${ }^{17}, \operatorname{Re} \chi^{(3)}(0 ; 0,0,0)=3.5 \times 10^{-19} \mathrm{~m}^{2} \mathrm{~V}^{-2}$, or the Kerr nonlinearity ${ }^{14}, \operatorname{Re} \chi^{(3)}(-\omega ; \omega,-\omega, \omega)=4 \times 10^{-19} \mathrm{~m}^{2} \mathrm{~V}^{-2}$. Using beam propagation techniques ${ }^{16}$ and our excitation conditions, we obtain a peak $\mathrm{THz}$ field at the detector of $\sim 10^{-1} \mathrm{~V} \mathrm{~m}^{-1}$ for a $2 \mu \mathrm{m}$ silicon film, much less than the $\sim 10 \mathrm{~V} \mathrm{~m}^{-1}$ measured here, suggesting that non-resonant rectification is weak. A further argument that the source currents are due to movement of electrons or holes comes from the dependence of the $\mathrm{THz}$ field strength on $\hbar \omega$ (see Fig. 2b). The solid curves indicate the expected dispersion, apart from a material-dependent scaling factor, of a nonlinear process proportional to the square root of single- and two-photon absorption coefficients expected for quantum interference between the absorption processes. These curves reproduce the experimental trends very well indicating that the $\mathrm{THz}$ emission is consistent with ballistically moving charge.

Although THz generation associated with interlevel, or bound carrier, transitions has been observed in doped silicon ${ }^{18}$, to the best of our knowledge, this is the first observation of $\mathrm{THz}$ generation from coherently generated free-carrier currents in clean, unbiased silicon on the basis of interband excitation. Measurements indicate that the $\mathrm{THz}$ field changes by less than $5 \%$ when the Si sample is rotated through $2 \pi$ about its normal with $\Delta \phi$ fixed. Currents were also generated with cross-polarized pulses. These currents are $\sim 3$ times smaller than those generated with collinearly polarized pulses, reflecting a weak anisotropy of the $\eta_{i j k l}$, as also predicted ${ }^{9}$ for 
a

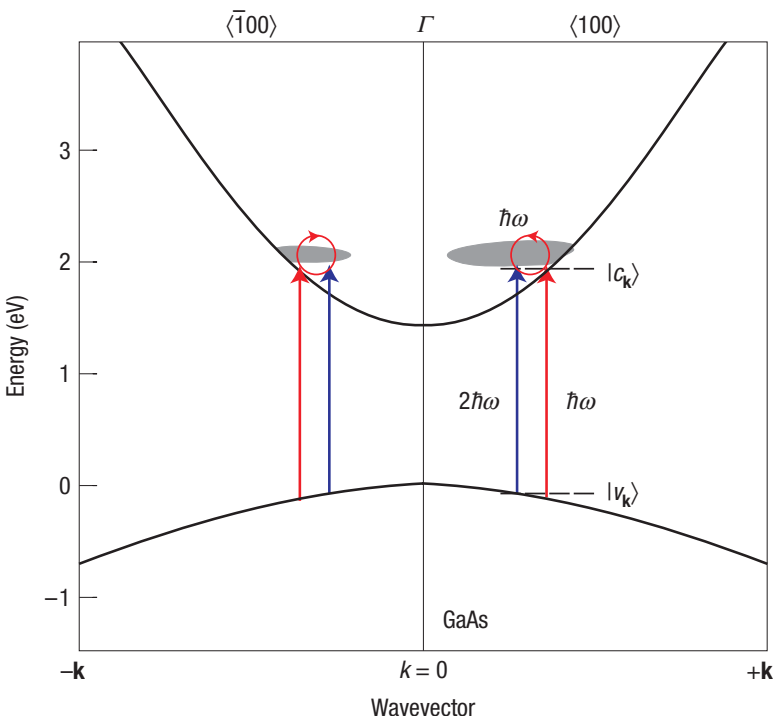

b

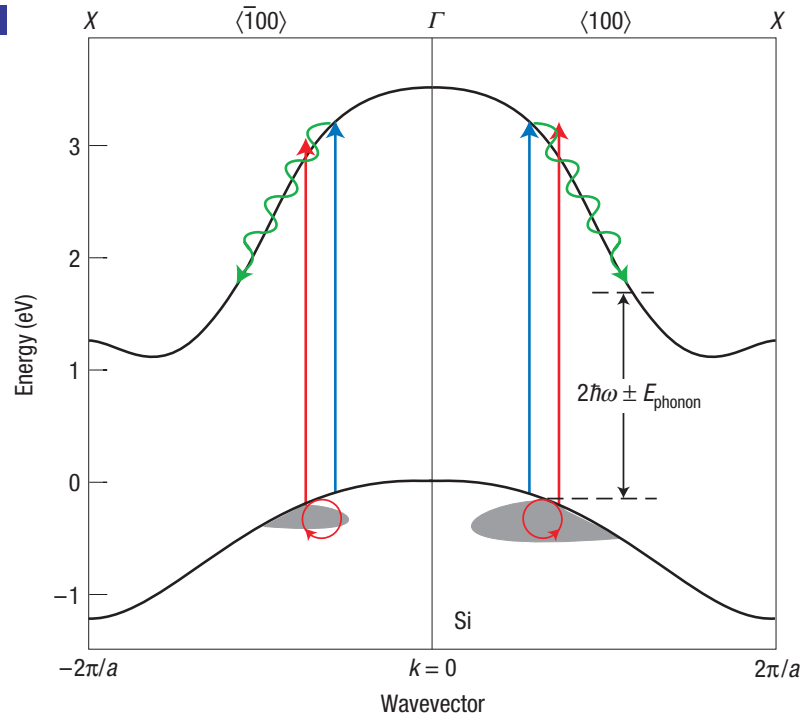

Figure 3 Schematic diagram of single- and two-photon quantum interference in GaAs and Si. a, Schematic energy-momentum band diagram of GaAs near the $\Gamma$ point showing one conduction and one valence band and two-colour excitation and interference across the fundamental bandgap; red is associated with the $\omega$ beam, blue with the $2 \omega$ beam. Asymmetric electron populations at $\pm \mathbf{k}$, and hence current generation, is indicated by shaded patches. $\mathbf{b}$, Corresponding band diagram for Si along the $\Gamma-X$ direction, showing interference for similar types of phonon-assisted transitions; phonon scattering with emission or absorption at energy $E_{\text {phonon }}$ is indicated.

GaAs. The peak THz field emitted by the GaAs or InP is $\sim 60$ times larger than in Si for identical optical excitation using $0.8 / 1.6 \mathrm{eV}$ pump pulses. However, given the difference in coherence lengths and absorption coefficients (for GaAs, the single- ${ }^{13}$ and two-photon absorption coefficients ${ }^{19}$ are an order of magnitude larger), the current density generated per electron-hole pair is only $\sim 7$ times larger in GaAs than in $\mathrm{Si}$. This difference might reflect a smaller scattering rate or smaller electron effective mass in GaAs versus Si.

Although a non-zero fourth-rank $\overleftrightarrow{\eta}$ tensor certainly is allowed in centrosymmetric media such as $\mathrm{Si}$, thereby permitting freecarrier current injection, the underlying microscopic mechanism is not obvious as Si has six conduction-band valleys and optical absorption requires phonon assistance from one of the six phonon branches. Given these complexities, current injection in $\mathrm{Si}$ is surprisingly efficient. A complete theoretical description is beyond the scope of this letter and here we only suggest how current injection might occur.

A comparison with GaAs, where theoretically and experimentally much more is known ${ }^{9,10}$, serves as a useful starting point for considering the current-injection mechanism in silicon. In GaAs, the quantum interference of single- and two-photon transition amplitudes, $a_{2 \omega}$ and $a_{\omega}$, which connects states with the same crystal momentum $\mathbf{k}$, leads to a polar distribution of electrons or holes. Optical transitions in solids can be understood as being induced by a minimal coupling perturbation hamiltonian $H \propto \mathbf{A} \cdot \mathbf{p} \propto \mathbf{E} \cdot \mathbf{p}$, where $\mathbf{A}$ and $\mathbf{E}$ are the vector potential and electric field associated with an optical plane wave and $\mathbf{p}$ is the momentum operator. For a direct-bandgap semiconductor such as GaAs with valence and conduction band states $\left|v_{\mathbf{k}}\right\rangle$ and $\left|c_{\mathbf{k}}\right\rangle$ at $\mathbf{k}, a_{2 \omega} \propto \mathbf{E}^{2 \omega} \cdot\left\langle v_{\mathbf{k}}|\mathbf{p}| \mathcal{c}_{\mathbf{k}}\right\rangle$. The $a_{\omega}$ requires intermediate states: in GaAs, the dominant intermediate states are the initial or final state. For example, if the conductionband state is the intermediate state, there is a contribution to $a_{\omega} \propto \mathbf{E}^{\omega} \mathbf{E}^{\omega}:\left\langle v_{\mathbf{k}}|\mathbf{p}| c_{\mathbf{k}}\right\rangle\left\langle c_{\mathbf{k}}|\mathbf{p}| c_{\mathbf{k}}\right\rangle \propto \mathbf{E}^{\omega} \mathbf{E}^{\omega}:\left\langle v_{\mathbf{k}}|\mathbf{p}| c_{\mathbf{k}}\right\rangle \mathbf{k}$. This is shown in Fig. 3a with the loop representing the 'self-scattering' term $\left\langle c_{\mathrm{k}}|\mathbf{p}| c_{\mathrm{k}}\right\rangle$. Interference between $a_{2 \omega}$ and $a_{\omega}$ therefore has a different sign for $\pm \mathbf{k}$, leading to a polar distribution of charge in valence or conduction bands; a charge current therefore flows. The sign and magnitude of the interference can be altered with the phase $\Delta \phi$. It is interesting to note that this self-scattering is fully equivalent to the field-induced acceleration in transport theories, where it has been shown to induce transition amplitudes that are odd in $k$-space ${ }^{20}$.

For silicon, the optically coupled states are constrained only by conservation of energy after phonon absorption/emission is taken into account. However, for current injection ${ }^{10}$, for the optical part of the transition the intermediate state should be the same as the initial or final state so that a self-scattering occurs (term in $\left.a_{\omega} \propto \pm \mathbf{k}\right)$ as in GaAs. One such process is shown in Fig. $3 \mathrm{~b}$ for initial states at $\pm \mathbf{k}$ along the $\langle 100\rangle$ and $\langle\overline{1} 00\rangle$ directions, and a selfscattering process in the valence band. However, for the process shown in Fig. $3 b$, the conduction-band states at $\pm k$ only serve as intermediate states within the coherent light-matter interaction involving photons as well as phonons. Phonon scattering can yield final states on opposite sides of a particular conduction-band valley with similar probabilities. Such states are associated with group velocities of opposite sign and thus the overall electron contribution to the current could be negligible. In contrast, the hole current is not affected by phonon scattering for the process shown in Fig. 3b. However, similar processes involving, for example, a selfscattering in the valence band, could also result in a non-negligible electron current. In GaAs, both electron and hole currents occur, but the electrons, because of their smaller effective mass, dominate the charge displacement. This difference could explain part of the discrepancy of the current magnitudes in GaAs and Si. However, given the relative complexity of, and number of pathways for the absorption processes in Si compared with those in GaAs, it is remarkable that a current is injected at all. Detailed theoretical and further experimental work is underway to elucidate the currentgeneration mechanism for silicon.

It is possible that strain or quantum confinement might improve the efficiency of the current-generation process or even allow more efficient current generation using a single-beam technique ${ }^{21}$ and a second-order nonlinear optical process. However, even for the third-order processes considered here, future studies 
can provide insight into how coherence control can lead to ballistic currents in Si and other indirect-bandgap semiconductors through phonon-assisted absorption.

\section{METHODS}

The optical source for the current-injection experiment is an optical parametric amplifier pumped with a $250 \mathrm{kHz}$ Ti:sapphire regenerative amplifier.

Second-harmonic pulses are obtained from a beta barium borate crystal. A piezoelectric transducer attached to one arm of the two-colour interferometer allows control of the relative phase of the pulses. Overlapped $\omega$ and $2 \omega$ pulses are focused to a $120 \mu \mathrm{m}$ spot size. Silicon samples with thicknesses of $0.5 \mu \mathrm{m}$, $2 \mu \mathrm{m}$ (silicon on sapphire), $125 \mu \mathrm{m}$ and $500 \mu \mathrm{m}$ (bulk silicon) were used. The low repetition rate of the experiment as well as the nonlinear optical processes involved in the infrared generation exclude effects related to carrier accumulation or possible influences of optical pre-pulses. The far-field $\mathrm{THz}$ emission perpendicular to the sample surface is analysed in a transmission geometry. The radiation is time-resolved using a $500-\mu \mathrm{m}$-thick $\mathrm{ZnTe}(110)$ electro-optic crystal with a detection bandwidth of $\sim 3 \mathrm{THz}$. Specifically, the induced birefringence for a $150 \mathrm{fs}, 810 \mathrm{~nm}(1.53 \mathrm{eV})$ probe beam is detected by a balanced detector scheme with a sensitivity of $\Delta I / I=1 \times 10^{-7} \mathrm{~Hz}^{-1 / 2}$, which allows for a quantitative analysis of the $\mathrm{THz}$ emission strength. The conversion of these field amplitudes to current densities relies on the comparison with current-injection studies in GaAs (ref. 16), taking into account the different $\mathrm{THz}$ emission strengths as well as the photoinjected carrier densities. For the data presented in Fig. 2a, the observed THz field strengths are corrected for the $P^{\omega} \sqrt{P^{2 \omega}}$ power scaling to account for the varying energies of the $\omega$ and $2 \omega$ pulses for different wavelengths. The simulated curves in Fig. $2 \mathrm{~b}$ are based on well-known linear absorption properties and theoretical results for the dispersion of the two-photon absorption coefficient above the direct bandgap ${ }^{22}$ for InP and GaAs or above the indirect gap of Si (ref. 15).

Received 29 December 2006; accepted 13 June 2007; published 8 July 2007.

\section{References}

1. Pavesi, L. \& Lockwood, D. J. Silicon Photonics (Springer, New York, 2004).

2. Rong, H. et al. A continuous-wave Raman silicon laser. Nature 433, 725-728 (2005).

3. Foster, M. A. et al. Broad-band optical parametric gain on a silicon photonic chip. Nature 441, 960-963 (2006).
4. Xu, Q., Schmidt, B., Pradham, S. \& Lipson, M. Micrometre-scale silicon electro-optic modulator. Nature 435, 235-237 (2005).

5. Jacobsen, R. S. et al. Strained silicon as a new electro-optic material. Nature 441, 199-202 (2006).

6. Rabitz, H., De Vivie-Riedle, R., Motzkus, M. \& Kompa, K. Whither the future of controlling quantum phenomena. Science 288, 824 (2000).

7. Hentschel, M. et al. Attosecond metrology. Nature 414, 509-513 (2001)

8. Xie, X., Dai, J. \& Zhang, X.-C. Coherent control of THz wave generation in air. Phys. Rev. Lett. 96 075005 (2006).

9. Atanasov, R. et al. Coherent control of photocurrent generation in bulk semiconductors. Phys. Rev. Lett. 76, 1703-1706 (1996).

10. Haché, A. et al. Observation of coherently controlled photocurrent in unbiased, bulk GaAs. Phys. Rev. Lett. 78, 306-309 (1997)

11. Bhat, R. D. R. \& Sipe, J. E. Optically injected spin currents in semiconductors. Phys. Rev. Lett. 85 , 5432-5435 (2000).

12. Stevens, M. J. et al. Optical injection and coherent control of a ballistic charge current in GaAs/AlGaAs quantum wells. Phys. Rev. Lett. 90, 136603 (2003).

13. Palik, E. D. Handbook of Optical Constants of Solids (Academic, New York, 1985).

14. Dinu, M. Dispersion of phonon-assisted nonresonant third-order nonlinearities. IEEE J. Quantum Electron. QE-39, 1498-1503 (2003).

15. Bristow, A. D. et al. Two photon absorption and Kerr coefficients of silicon for 850-2200 nm. Appl. Phys. Lett. 90, 191104 (2007).

16. Côté, D. et al. Simple method for calculating the propagation of terahertz radiation in experimental geometries. J. Opt. Soc. Am. B 20, 1374-1385 (2003).

17. Moss, D. J. et al. Band-structure calculation of dispersion and anisotropy in $\chi^{(3)}$ for 3rd harmonic generation in Si, Ge and GaAs. Phys. Rev. B 41, 1542-1560 (1990).

18. Hübers, H.-W., Pavlov, S. G. \& Shastin, V. N. Terahertz lasers based on germanium and silicon. Semicond. Sci. Technol. 20, S211-S221 (2005).

19. Dworak, M. D. et al. Measurement of the anisotropy of two-photon absorption coefficients in zincblende semiconductors. IEEE J. Quantum Electron. 30, 256-268 (1994).

20. Duc, H. T. et al. Temporal decay of coherently optically injected charge and spin currents due to carrier-LO-phonon and carrier-carrier scattering. Phys. Rev. B 74, 165328 (2006).

21. Laman, N., Shkrebtii, A. I., Sipe, J. E. \& van Driel, H. M. Quantum interference control of currents in CdSe with a single optical beam. Appl. Phys. Lett. 75, 2581-2583 (1999).

22. Sheik-Bahae, M. et al. Dispersion of bound electron nonlinear refraction in solids. IEEE J. Quantum 2. Sheik-Bahae, M. et al. Dispersio
Electron. 27, 1296-1309 (1991).

\section{Acknowledgements}

We gratefully acknowledge stimulating discussions with J. E. Sipe and A. L. Smirl. The financial support of the Natural Sciences and Engineering Research Council of Canada is gratefully appreciated. M.B. is also appreciative of support from the Alexander von Humboldt Foundation. Correspondence and requests for materials should be addressed to H.M.v.D.

Author contributions

H.M.v.D. suggested the experiments. M.B., M.S., L.C. and A.D.B. designed the experiments and carried out measurements. All authors contributed in discussions and analysis.

Competing financial interests

The authors declare no competing financial interests.

Reprints and permission information is available online at http://npg.nature.com/reprintsandpermissions/ 